\title{
Solvents for sustainable chemical processes
}

\author{
P. Pollet ${ }^{1,2}$, C. A. Eckert ${ }^{1,2,3}$ \& C. L. Liotta ${ }^{1,2,3}$ \\ ${ }^{1}$ School of Chemistry \& Biochemistry, \\ Georgia Institute of Technology, USA \\ ${ }^{2}$ Specialty Separations Center, Georgia Institute of Technology, USA \\ ${ }^{3}$ School of Chemical \& Biomolecular Engineering, \\ Georgia Institute of Technology, USA
}

\begin{abstract}
Worldwide, socioeconomic strategies encourage - and may soon enforce industrial sustainability. Therefore, innovative research and approaches must provide short and long term solutions to reach future legislative targets. For chemical processes, the development of solvents that facilitate reaction and subsequent product separation is paramount to secure economic competitivity while minimizing impact on the environment and energy consumption. Tunable and switchable solvents were developed to address synergistically reaction and separation. Tunable solvents change properties continuously upon application of an external stimulus. For example, organic aqueous solvents allow for the reaction to be homogeneously catalyzed followed by a simple and efficient heterogeneous separation of the product (commonly a heterogeneous catalysis attribute). Chemical processes mediated by water at or below the near critical range can also alleviate the shortcomings of current synthetic strategies (waste production and management, remediation costs). In contrast, switchable solvents change physical properties abruptly upon application of an external stimulus. Piperylene sulfone is one example and can provide a recyclable alternative for dipolar, aprotic solvents such as dimethylsulfoxide (DMSO). Solvents can accomplish more than heat and mass transfer; they can actively contribute to facilitate reaction and product separation while minimizing waste generation and energy consumption.
\end{abstract}

Keywords: solvents, sustainability, reaction, separation, smart solvents, switchable, tunable, near critical water, piperylene sulfone, OATS. 


\section{Introduction}

The solvents discussed here provide simple and efficient vehicles for conducting reactions and separations. This is accomplished by exposing the solvent system to an external physical or chemical stimulus which results in a dramatic change in its physical and chemical properties. Tunable solvents are defined as solvents that change properties continuously upon application of an external stimulus. Supercritrical fluids (SFC), (Dillow et al. [1], Brown et al. [2], Thompson et al. [3], Brown et al. [4], Nolen et al. [5], Eckert et al. [6], Koch et al. [7], Furstner et al. [8], Solinas et al. [9], Maayan et al. [10]) nearcritical liquids (Eckert et al. [6], Chandler et al. [11], Chandler et al. [12], Lesutis et al. [13], Patrick et al. [14], Nolen et al. [15]) and gas-expanded liquids (GXLs) (Jessop and Subramaniam [16], Ablan et al. [17], Jessop et al. [18]) are examples of tunable solvents. It has been demonstrated that these solvents systems are useful for coupling reactions and separations. (Dillow et al. [1], Eckert et al. [6], Daintree et al. [19], Eckert et al. [20], Eckert and Chamber [21], Eckert et al. [22, 23], Jessop [24], Ramsey et al. [25], Rayner [26], Rezaei et al. [27], Tang et al. [28]) In contrast, switchable solvents are solvents that change physical properties abruptly. In other words, they can be switched "on" and "off". This unique property is a consequence of a reversible reaction (i.e. addition/elimination reactions) in response to an external stimulus such as adjusting temperature and/or the addition or removal of a gas. Because of the reversibility of the reaction, the changed solvent can be brought back to its original state.

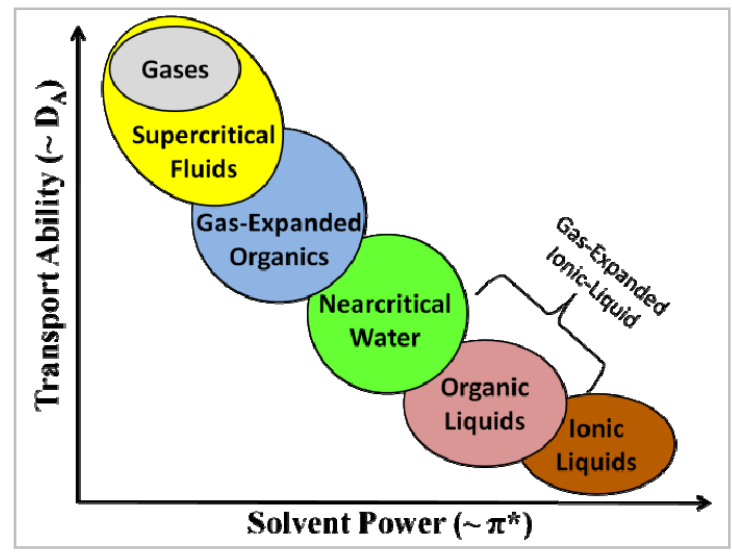

Figure 1: $\quad$ Solvent power and transport ability of various solvents.

Figure 1 relates qualitatively the solvent power (in terms of the Kamlet-Taft polarizability/dipolarity, $\pi^{*}$ ) to transport ability (in terms of diffusion coefficient, $\mathrm{D}_{\mathrm{A}}$ ) for the relevant solvents. Most liquids are strong solvents but they have low diffusion coefficients (about four to six orders of magnitude smaller than those for gases, Hines and Maddox [29], Bird et al. [30]) which may lead to mass transfer limitations. In contrast, gases have far better transport properties but are 
much weaker solvents. Supercritical fluids such as $\mathrm{CO}_{2}$ are much stronger solvents than gaseous $\mathrm{CO}_{2}$ and have much high diffusion coefficients than liquids. Gas-expanded liquids (GXLs), mixtures of organics and dissolved gases, are stronger solvents than supercritical fluids and have better diffusion coefficients than liquids. For any given process, the choice of solvent system depends on the physical and chemical properties of the reactants and products, the processing requirements, and environmental considerations.

It is important to develop reaction processes in a holistic manner. Just having a high yield reaction is not enough. The ease of separation of the desired product from the reaction system is also of paramount importance. As a consequence, our focus is placed on solvent systems that combine the benefits of homogeneous reactions and heterogeneous separations. Homogeneous reactions are usually superior to heterogeneous reactions in terms of reaction rates, process control, and selectivity. However, the difficulty of separating and recycling the catalyst limits economic applications of homogeneous catalysis. Heterogeneous catalysis is widely used industrially since it has a built-in separation of the product from the catalyst. The alternative solvent systems combine homogeneous reactions with heterogeneous separations. Herein, we discuss applications benefiting from the use of tunable and switchable solvents.

\section{Tunable solvents}

\subsection{Organic aqueous tunable solvent systems (OATS)}

Organic Aqueous Tunable Solvent (OATS) are homogeneous mixtures of aprotic organics (acetonitrile or tetrahydrofuran) and polar protic solvents (water or polyethylene glycol) that undergo a phase split to form biphasic liquid-liquid mixtures upon the addition of an antisolvent gas. The phase splitting - going from monophasic to biphasic - results from the difference in the antisolvent gas solubility between the aprotic organic solvent and the polar protic solvent. $\mathrm{CO}_{2}$ is completely miscible with most organics but has only slightly solubility in aqueous media, and, as a consequence, is an effective antisolvent in promoting phase splitting. The resulting biphasic system consists of a GXL and a polar liquid phase. The physical properties of GXLs are readily tuned by pressure. In most catalyzed reactions, it is imperative to recover and recycle the expensive catalyst. OATS provide a simple and efficient method for separating hydrophilic catalysts from organophilic substrates. They offer increased reaction rates and improved yields and selectivity - often seen in homogeneous systems - as well as simple, efficient separation (characteristic of heterogeneous separation) and recycle of the catalyst. As a demonstration, we conducted hydroformylations of hydrophobic 1-octene and $p$-methylstyrene in $\mathrm{THF} / \mathrm{H}_{2} \mathrm{O}$ and $\mathrm{ACN} / \mathrm{H}_{2} \mathrm{O}$, respectively, under syngas pressure (mole ratio of $1: 1 \mathrm{CO}: \mathrm{H}_{2}$ ) to produce the corresponding branched and linear aldehydes in the presence of rhodium catalyst (Rh) with triphenylphosphine (TPP) ligands. Hydroformylation reactions are carried out at pressures of about $3 \mathrm{MPa}$ (Blasucci et al. [31], El Ali et al. [32], Hallett et al. [33], Nair et al. [34]) and therefore; the use of $\mathrm{CO}_{2}$ pressures 
(around 3.5 $\mathrm{MPa}$ ) with the OATS does not require equipment modifications. Industrial hydroformylations are run in biphasic aqueous-organic systems, where the reaction occurs in the aqueous phase in the presence of the water-soluble sulfonated Rh/TPP catalyst and the products are extracted into the organic phase. As a consequence, this approach is practical only for water-soluble alkenes (mainly C3 and C4). OATS permits the hydroformylation of virtually insoluble alkenes such as 1-octene and p-methylstyrene to form the corresponding aldehydes. For example, we reported the hydroformylation of $p$-methylstyrene to produce 2-( $p$-tolyl)propanal (branched product) and 3-( $p$-tolyl)propanal in $\mathrm{ACN} / \mathrm{H}_{2} \mathrm{O}(70 \mathrm{vol} \%$ ACN) OATS (Figure 2). The branched aldehyde - the desired product - is a model for the intermediate (2-(4-isobutylphenyl)propanal) in the ibuprofen synthesis. The TOF was 92 at $40^{\circ} \mathrm{C}$ and 406 at $80^{\circ} \mathrm{C}$ and the yield of the branched product was $95 \%$ at $40^{\circ} \mathrm{C}$ and $80 \%$ at $80^{\circ} \mathrm{C}$. The reduction in the yield of the branched product with temperature is attributed to $\beta$-hydride elimination,(Nair et al. [34]) where the intermediate complex of the catalyst with the branched product is converted back into the starting material at higher temperatures. The styrene conversion rates in OATS show at least an order of magnitude improvement over heterogeneously reported systems using solid supports and reactions run with ionic liquid modified silica sol-gel. Also, the branched product selectivity improves by more than $30 \%$. The separation of the aldehyde is outstanding; at $2.5 \mathrm{MPa}$ of $\mathrm{CO}_{2}$ pressure, more than $99 \%$ of the branched aldehyde product partitions in the acetonitrile-rich phase.
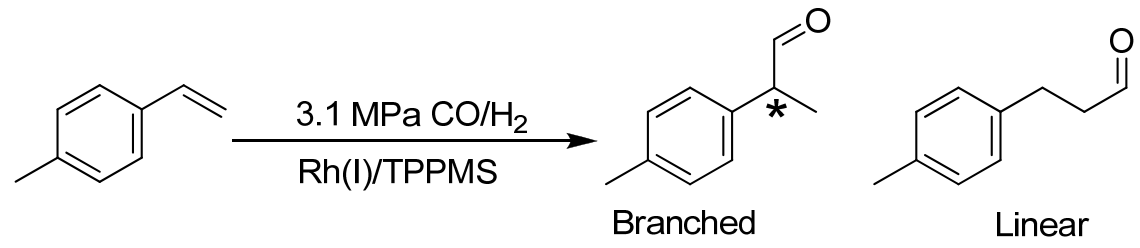

Figure 2: Hydroformylation of p-methylstyrene with $\mathrm{Rh} / \mathrm{TPPMS}$ to produce branched (desired) and linear aldehydes.

\subsection{Near critical water}

Near critical water $(\mathrm{NCW})$ is hot liquid water (critical point: $374^{\circ} \mathrm{C}, 22.1 \mathrm{MPa}$ ) at temperatures between $200-350^{\circ} \mathrm{C}$. At near critical conditions, water has physical properties similar to acetone. At $275^{\circ} \mathrm{C}$, the density drops to $0.7-0.8$ and the dielectric constant decreases to 20 at $275^{\circ} \mathrm{C}$. This is mostly due to diminished hydrogen bonding. The solubility of nonpolar organics is markedly improved in NCW while it remains an effective medium for dissolving ionic compounds. Thus, it becomes possible to conduct reactions between hydrophobic organic molecules and hydrophilic ionic reactions under homogeneous conditions at the near critical temperature range and then, subsequently, easily isolate the organic product at room temperature upon phase split. Additionally, the dissociation constant of water $\mathrm{K}_{\mathrm{W}}$ increases gradually from $10^{-14}$ at $25^{\circ} \mathrm{C}$ to about $10^{-11}$ at $275^{\circ} \mathrm{C}$. This increase in $\mathrm{K}_{\mathrm{W}}$ offers opportunities for acid or base catalysis, 
avoiding the need for added acids and bases and the subsequent waste-intensive neutralization steps.

We reported the Lewis acid-free Friedel-Crafts alkylation of phenol with tertbutyl alcohol in water at $275^{\circ} \mathrm{C}$, as shown in Figure 3 (Chandler et al. [11]). Traditionally, these reactions use aluminum chloride $\left(\mathrm{AlCl}_{3}\right)$, which is corrosive, water-sensitive, difficult to handle, and often used in greater than stoichiometric quantities - leading to substantial waste generation. The yield of 2-tertbutylphenol reached $17 \mathrm{~mol} \%$ after 30 hours of reaction time and then decreases to an equilibrium concentration of $10 \%$ and the yield of 4-tert-butylphenol increased linearly to $20 \%$ after 50 hours of reaction time. The 2-tert-butylphenol and 4-tert-butylphenol yields in NCW are similar to acid-catalyzed reactions reported in the literature with the use of five times excess of $\mathrm{HClO}_{4}$. $\mathrm{NCW}$ plays a dual role as solvent and catalyst. Thus, NCW for Friedel-Crafts acylations could eliminate the use of mineral or Lewis acids and eliminate the subsequent neutralization steps that generate large quantities of waste salt for each pound of products.

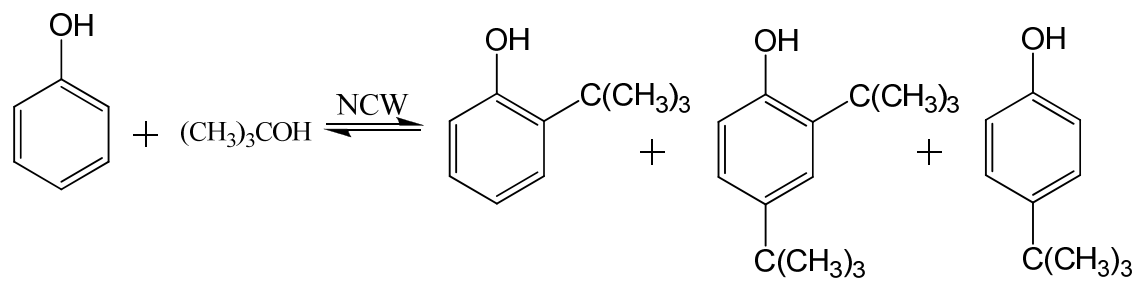

Figure 3: Alkylation of phenol with tert-butanol to produce 2-tertbutylphenol, 2,4-di-tert-butylphenol, and 4-tert-butylphenol.

\section{Switchable solvents}

\subsection{Piperylene sulfone}

Piperylene sulfone is synthesized from trans-1,3-pentadiene (trans-piperylene) and sulfur dioxide in the presence of a radical inhibitor (such as N-phenyl-2naphtylamine). The synthesis can also be carried out using the commercially available and inexpensive 1,3-pentadiene, which is a mixture of trans- and cisisomers. It should be noted that the cis-isomer reacts very slowly, if at all, with $\mathrm{SO}_{2}$ to form the cyclic sulfone. We measured and reported the solvatochromic properties of piperylene sulfone, showing that piperylene sulfone has comparable solvent properties to dimethyl sulfoxide (DMSO, Vinci et al. [35]). The important difference between piperylene sulfone and DMSO is that the former is switchable and the latter is not. Indeed, at temperatures greater than $100^{\circ} \mathrm{C}$ piperylene sulfone cleanly and efficiently decomposes to gaseous trans-1,3pentadiene (b.p. $42^{\circ} \mathrm{C}$ ) and sulfur dioxide (b.p. $-10^{\circ} \mathrm{C}$ ) via a retro-cheletropic process (Figure 4). The products can be collected and allowed to react to reform the piperylene sulfone solvent. 


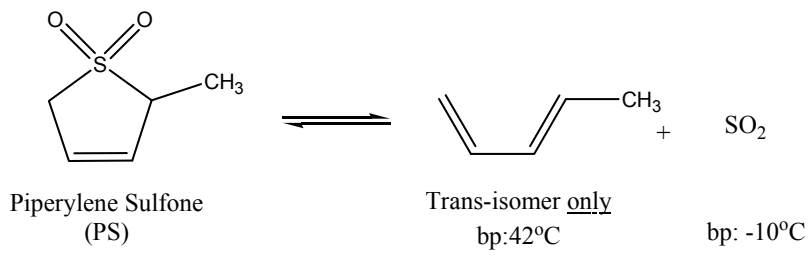

Figure 4: Reversible decomposition of piperylene sulfone into transpiperylene and sulfur dioxide.

Critical to employing piperylene sulfone as a solvent for coupling reactions and separations is the requirement that decomposition of the solvent occurs with $100 \%$ efficiency. Differential scanning calorimetry coupled with thermogravimetric analysis was performed on piperylene sulfone. Heating at $5^{\circ} \mathrm{C} / \mathrm{min}$ for 20 minutes and then holding at $120^{\circ} \mathrm{C}$ for 30 minutes triggered the decomposition, leaving no residual mass. Thus, in principle, facile product recovery and solvent recycle can be realized. Piperylene sulfone is one of a number of possible $\beta, \gamma$-unsaturated cyclic sulfones (also called sulfolenes) that can be employed as solvents. Others sulfolenes such as butadiene sulfone and isoprene sulfone are also of interest for switchable solvents applications. We reported the rates of substitution reaction of benzyl chloride with various anionic nucleophiles (Figure 5) in both piperylene sulfone and DMSO at $40^{\circ} \mathrm{C}$ (Vinci et al. [35]). The rate constants are slightly greater in PS than in DMSO for the reaction of benzyl chloride with KSCN with values of $2.1 \pm 0.1$ and $1.4 \pm 0.1 \mathrm{~L} / \mathrm{mol}$.s, respectively. The rate constants were similar for the reaction with potassium thioacetate and pyrrolidine-dithiocarbamate. The addition of small amounts of water to the reaction mixtures improves the rate in both PS and DMSO for the reaction of benzyl chloride with $\mathrm{KOAc}, \mathrm{KCN}$, and $\mathrm{KSCN}$. This improvement could result from the improved solubility of the reacting salts. After reaction, the mixtures were heated to $110^{\circ} \mathrm{C}$ to decompose PS to gaseous trans-piperylene and sulfur dioxide. The solvent-free benzyl thiocyanate residue was isolated with $96 \%$ yield. The gases were collected and PS was reformed with $87 \%$ yield. The small size of the experimental set up is believed to be the reason for the loss of PS; greater solvent recovery yields are expected on larger scale.

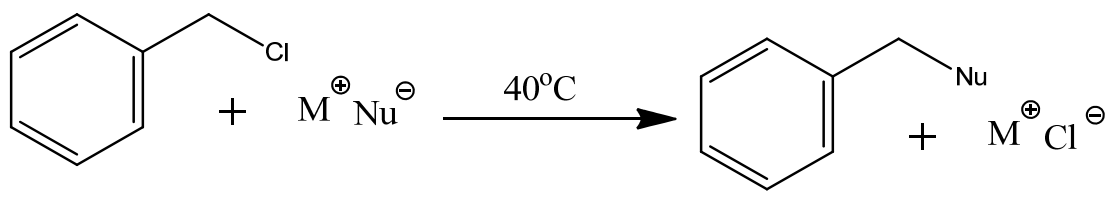

Figure 5: Nucleophilic displacement reactions.

\subsection{Reversible ionic liquids (RevILs)}

A reversible ionic liquid is a solvent system which can switch back and forth between a medium which is ionic and a medium which is non-ionic; the latter is referred to as a molecular medium. There are two major classes of reversible 
ionic liquids (RevILs): two-component and one-component. Two-component RevILs are based on an equimolar mixture of a neutral alcohol and a neutral molecule containing at least one basic nitrogen functionality. Examples of the latter are 1,8-diazabicyclo[5.4.0]undec-7-ene (DBU) and 2-butyl-1,1,3,3tetramethylguanidine (TMBG, Phan et al. [36], Jessop et al. [37], Heldebrant et al. [38]). One-component systems eliminate the need for an alcohol as seen in the two-component RevILs; these systems employ solely a neutral molecule containing at least one basic nitrogen functionality [12]. An example of a onecomponent system are the silylated amines such as trialkoxy- or trialkylsilylpropylamines as molecular liquid. Upon reaction with $\mathrm{CO}_{2}$, these amine precursors form ionic liquids composed of the corresponding carbamate anion and ammonium cation pairs (Figure 6). The Lewis acid character of the silicon substitutent is believed to play a role in controlling the temperatures at which carbon dioxide is captured and released.

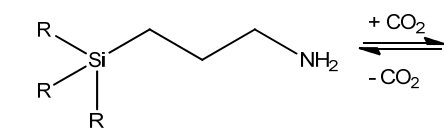

R: alkyl or alkoxy group

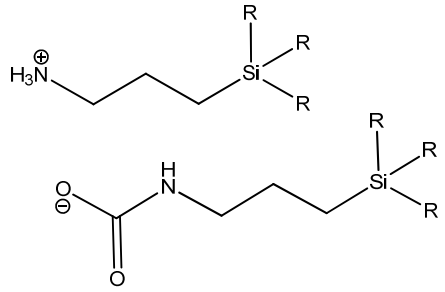

Ionic Liquid

Figure 6: One-component system: reversible switch from a molecular liquid trialkoxy and trialkylsilylpropylamine to its corresponding ionic liquid upon addition of $\mathrm{CO}_{2}$.

\subsection{The Claisen-Schmidt condensation}

The Claisen-Schmidt condensation of butanone and benzaldehyde in RevIL system (TMBG/MeOH) was reported by Hart et al. [39] (Figure 7). Three products are formed upon reaction: the internal enone (3-methyl-4-phenyl-but-3en-2-one), the terminal enone (1-phenyl-pent-1-en-3-one) and water. Under basic conditions, the terminal enone product is the predominant product formed. It should noted that TMBG plays the dual role of base catalyst and solvent.<smiles>CCC(=O)/C=C/c1ccccc1</smiles>

Figure 7: Claisen-Schmidt condensation of 2-butanone and benzaldehyde. 
After 24 hours at room temperature or 3 hours at $80^{\circ} \mathrm{C}$, yields of $48 \%$ and $44 \%$ in enone products were obtained, respectively. The formation of the terminal and internal enone-products was also studied as a function of time at $80^{\circ} \mathrm{C}$. As the reaction time increased, the yields in enone products first increased from $13 \%$ at 1 hour to $44 \%$ at 3 hours and then began to decrease after 4 hours. The decrease was attributed to competing condensation processes between the enone products and the benzaldehyde, resulting in lower overall yields of the desired products. As a consequence, shorter reaction times to partial conversions were necessary in order to develop a process in which isolated yields were maximized and solvent recycle was possible. The isolation of the enone products was performed by adding n-octane and methanol to the reaction mixture, followed by the addition of $\mathrm{CO}_{2}$, which triggered the formation of the ionic liquid. Under these conditions an octane phase separated from the newly developed ionic liquid phase. The enone products were predominantly soluble in the octane phase and were easily separated by decantation.

\section{4 $\mathrm{CO}_{2}$ capture}

RevILs are also being studied for absorbing $\mathrm{CO}_{2}$ from various flue gas streams, where they may require less energy for $\mathrm{CO}_{2}$ capture and release than the conventional alkanolamine process. The presence of large volumes of water (commonly $70 \mathrm{vol} \%$ ) increases the energy demand for the thermal regeneration of the aqueous alkanolamines. RevILs behave in a dual capacity as solvents for $\mathrm{CO}_{2}$ capture: (1) the chemisorption of $\mathrm{CO}_{2}$ upon reaction of the molecular liquid to form RevIL and (2) by the physical absorption of the $\mathrm{CO}_{2}$. We reported the use of trimethoxy (TMSA), triethoxy (TESA), triethyl (TEtSA), and tripropyl (TPSA) silated propylamines for $\mathrm{CO}_{2}$ capture (Blasucci et al. [40, 41]). The chemical capacities can be enhanced by the physisorption capabilities of the RevILs. The physisorption is a much less exothermic process, thus the regeneration of physisorbed $\mathrm{CO}_{2}$ requires less energy.

\section{Conclusions}

Solvents are necessary for most chemical processes and lead to a large percentage of the process cost and to the magnitude of the waste-stream. In order to achieve chemical processes that are both competitive and environmentally conscious, solvents must address simultaneously reaction efficiency, product separation, and recyclability. Tunable solvents and smart switchable solvents can improve specific families of chemical reactions and processes. These solvents change properties upon application of physical or chemical stimuli and, as a consequence, provide facile separation techniques. Smart switchable solvents change properties abruptly and reversibly. Unlike DMSO, because of a facile and reversible reaction between trans-piperylene and sulfur dioxide, piperylene sulfone allows for easy product isolation and solvent recycle are easily accomplished. In addition, Claisen-Schmidt condensation was successfully carried out in RevILs with the added capabilities of improved 
separation of the product and recycling of the solvent system. Reversible ionic liquids facilitate catalyst and product recovery, yielding efficient separations of catalysts for reuse. Each of these different solvents has advantages and limitations. The feasibility of their applications depend on the specifics of each reaction or process. Importantly, it should be recognised that solvents can accomplish more than heat and mass transfer; they can actively contribute to facilitate reaction and product separation while minimizing waste generation.

\section{References}

[1] Dillow, A.K., Brown, J.S., Liotta, C.L., Eckert, C.A., Supercritical fluid tuning of reactions rates: The cis-trans isomerization of 4-4 '-disubstituted azobenzenes, J Phys Chem A, 102, 7609-7617, 1998.

[2] Brown, J.S., Lesutis, H.P., Lamb, D.R., Bush, D., Chandler, K., West, B.L., Liotta, C.L., Eckert, C.A., Schiraldi, D., Hurley, J.S., Supercritical fluid separation for selective quaternary ammonium salt promoted esterification of terephthalic acid, Ind Eng Chem Res, 38, 3622-3627, 1999.

[3] Thompson, R.L., Glaser, R., Bush, D., Liotta, C.L., Eckert, C.A., Rate variations of a hetero-Diels-Alder reaction in supercritical fluid $\mathrm{CO} 2$, Ind Eng Chem Res, 38, 4220-4225, 1999.

[4] Brown, R.A., Pollet, P., McKoon, E., Eckert, C.A., Liotta, C.L., Jessop, P.G., Asymmetric hydrogenation and catalyst recycling using ionic liquid and supercritical carbon dioxide, J Am Chem Soc, 123, 1254-1255, 2001.

[5] Nolen, S.A., Lu, J., Brown, J.S., Pollet, P., Eason, B.C., Griffith, K.N., Glaser, R., Bush, D., Lamb, D.R., Liotta, C.L., Eckert, C.A., Thiele, G.F., Bartels, K.A., Olefin epoxidations using supercritical carbon dioxide and hydrogen peroxide without added metallic catalysts or peroxy acids, Ind Eng Chem Res, 41, 316-323, 2002.

[6] Eckert, C.A., Liotta, C.L., Bush, D., Brown, J.S., Hallett, J.P., Sustainable reactions in tunable solvents, J Phys Chem B, 108, 18108-18118, 2004.

[7] Koch, D., Leitner, W., Rhodium-catalyzed hydroformylation in supercritical carbon dioxide, J Am Chem Soc, 120, 13398-13404, 1998.

[8] Furstner, A., Ackermann, L., Beck, K., Hori, H., Koch, D., Langemann, K., Liebl, M., Six, C., Leitner, W., Olefin metathesis in supercritical carbon dioxide, J Am Chem Soc, 123, 9000-9006, 2001.

[9] Solinas, M., Jiang, J.Y., Stelzer, O., Leitner, W., A cartridge system for organometallic catalysis: Sequential catalysis and separation using supercritical carbon dioxide to switch phases, Angew Chem Int Edit, 44, 2291-2295, 2005.

[10] Maayan, G., Ganchegui, B., Leitner, W., Neumann, R., Selective aerobic oxidation in supercritical carbon dioxide catalyzed by the H5PV2Mo10O40 polyoxometalate, Chem Commun, 2230-2232, 2006.

[11] Chandler, K., Deng, F.H., Dillow, A.K., Liotta, C.L., Eckert, C.A., Alkylation reactions in near-critical water in the absence of acid catalysts, Ind Eng Chem Res, 36, 5175-5179, 1997. 
[12] Chandler, K., Liotta, C.L., Eckert, C.A., Schiraldi, D., Tuning alkylation reactions with temperature in near-critical water, Aiche J, 44, 2080-2087, 1998.

[13] Lesutis, H.P., Glaser, R., Liotta, C.L., Eckert, C.A., Acid/base-catalyzed ester hydrolysis in near-critical water, Chem Commun, 2063-2064, 1999.

[14] Patrick, H.R., Griffith, K., Liotta, C.L., Eckert, C.A., Glaser, R., Near-critical water: A benign medium for catalytic reactions, Ind Eng Chem Res, 40, 6063-6067, 2001.

[15] Nolen, S.A., Liotta, C.L., Eckert, C.A., Glaser, R., The catalytic opportunities of near-critical water: a benign medium for conventionally acid and base catalyzed condensations for organic synthesis, Green Chem, 5, 663-669, 2003.

[16] Jessop, P.G., Subramaniam, B., Gas-expanded liquids, Chem Rev, 107, 2666-2694, 2007.

[17] Ablan, C.D., Hallett, J.P., West, K.N., Jones, R.S., Eckert, C.A., Liotta, C.L., Jessop, P.G., Use and recovery of a homogeneous catalyst with carbon dioxide as a solubility switch, Chem Commun, 2972-2973, 2003.

[18] Jessop, P.G., Stanley, R.R., Brown, R.A., Eckert, C.A., Liotta, C.L., Ngo, T.T., Pollet, P., Neoteric solvents for asymmetric hydrogenation: supercritical fluids, ionic liquids, and expanded ionic liquids, Green Chem, 5, 123-128, 2003.

[19] Daintree, L.S., Kordikowski, A., York, P., Separation processes for organic molecules using SCF technologies, Advanced Drug Delivery Reviews, 60, 351-372, 2008.

[20] Eckert, C.A., Bush, D., Brown, J.S., Liotta, C.L., Tuning solvents for sustainable technology, Industrial \& Engineering Chemistry Research, 39, 4615-4621, 2000.

[21] Eckert, C.A., Chandler, K., Tuning fluid solvents for chemical reactions, J Supercrit Fluid, 13, 187-195, 1998.

[22] Eckert, C.A., Knutson, B.L., Debenedetti, P.G., Supercritical fluids as solvents for chemical and materials processing, Nature, 383, 313-318, 1996.

[23] Eckert, C.A., Vanalsten, J.G., Stoicos, T., Supercritical Fluid Processing, Environ Sci Technol, 20, 319-325, 1986.

[24] Jessop, P.G., Homogeneous catalysis using supercritical fluids: Recent trends and systems studied, Journal of Supercritical Fluids, 38, 211-231, 2006.

[25] Ramsey, E., Sun, Q.B., Zhang, Z.Q., Zhang, C.M., Gou, W., Mini-Review: Green sustainable processes using supercritical fluid carbon dioxide, Journal of Environmental Sciences-China, 21, 720-726, 2009.

[26] Rayner, C.M., The potential of carbon dioxide in synthetic organic chemistry, Organic Process Research \& Development, 11, 121-132, 2007.

[27] Rezaei, K., Temelli, F., Jenab, E., Effects of pressure and temperature on enzymatic reactions in supercritical fluids, Biotechnology Advances, 25, 272-280, 2007. 
[28] Tang, Z., Xie, W.H., Zong, B.N., Min, E.Z., Recent research progress of chemical reactions under supercritical conditions, Chinese Journal of Chemical Engineering, 12, 498-504, 2004.

[29] Hines, A.L., Maddox, R.N., Mass Transfer Fundamentals and Applications Prentice Hall, New Jersey, 1985.

[30] Bird, R.B., Stewart, W.E., Lightfoot, E.N., Transport Phenomena, Second ed., Wiley India, New Delhi, 2006.

[31] Blasucci, V.M., Husain, Z.A., Fadhel, A.Z., Donaldson, M.E., Vyhmeister, E., Pollet, P., Liotta, C.L., Eckert, C.A., Combining Homogeneous Catalysis with Heterogeneous Separation using Tunable Solvent Systems, Journal of Physical Chemistry A, 114, 3932-3938, 2010.

[32] El Ali, B., Tijani, J., Fettouhi, M., El-Faer, M., Al-Arfaj, A., Rhodium(I) and rhodium(III)-heteropolyacids supported on MCM-41 for the catalytic hydroformylation of styrene derivatives, Appl Catal a-Gen, 283, 185-196, 2005.

[33] Hallett, J.P., Ford, J.W., Jones, R.S., Pollet, P., Thomas, C.A., Liotta, C.L., Eckert, C.A., Hydroformylation catalyst recycle with gas-expanded liquids, Industrial \& Engineering Chemistry Research, 47, 2585-2589, 2008.

[34] Nair, V.S., Mathew, S.P., Chaudhari, R.V., Kinetics of hydroformylation of styrene using homogeneous rhodium complex catalyst, Journal of Molecular Catalysis a-Chemical, 143, 99-110, 1999.

[35] Vinci, D., Donaldson, M., Hallett, J.P., John, E.A., Pollet, P., Thomas, C.A., Grilly, J.D., Jessop, P.G., Liotta, C.L., Eckert, C.A., Piperylene sulfone: a labile and recyclable DMSO substitute, Chem Commun, 1427-1429, 2007.

[36] Phan, L., Chiu, D., Heldebrant, D.J., Huttenhower, H., John, E., Li, X.W., Pollet, P., Wang, R.Y., Eckert, C.A., Liotta, C.L., Jessop, P.G., Switchable solvents consisting of amidine/alcohol or guanidine/alcohol mixtures, Ind Eng Chem Res, 47, 539-545, 2008.

[37] Jessop, P.G., Heldebrant, D.J., Li, X.W., Eckert, C.A., Liotta, C.L., Green chemistry - Reversible nonpolar-to-polar solvent, Nature, 436, 1102-1102, 2005.

[38] Heldebrant, D.J., Jessop, P.G., Thomas, C.A., Eckert, C.A., Liotta, C.L., The reaction of 1,8-diazabicyclo[5.4.0] undec-7-ene (DBU) with carbon dioxide, J Org Chem, 70, 5335-5338, 2005.

[39] Hart, R., Pollet, P., Hahne, D.J., John, E., Llopis-Mestre, V., Blasucci, V., Huttenhower, H., Leitner, W., Eckert, C.A., Liotta, C.L., Benign coupling of reactions and separations with reversible ionic liquids, Tetrahedron, 66, 1082-1090, 2010.

[40] Blasucci, V., Hart, R., Mestre, V.L., Hahne, D.J., Burlager, M., Huttenhower, H., Thio, B.J.R., Pollet, P., Liotta, C.L., Eckert, C.A., Single component, reversible ionic liquids for energy applications, Fuel, 89, 1315-1319, 2010.

[41] Blasucci, V.M., Hart, R., Pollet, P., Liotta, C.L., Eckert, C.A., Reversible ionic liquids designed for facile separations, Fluid Phase Equilibria, 294, $1-6,2010$. 